\title{
WebSIG alimentado com dados coletados com sistema de mapeamento móvel terrestre
}

WebSIG built with data collected with terrestrial Mobile Mapping System

\author{
Rodrigo Bezerra de Araújo Gallis ${ }^{1}$ \\ Ricardo Luís Barbosa ${ }^{1}$ \\ Samuel Rodrigues Cunha ${ }^{1}$ \\ Alan Kazuo Hiraga ${ }^{2}$ \\ Marcelo Araújo Faria ${ }^{1}$
}

Recebido em abril de 2018.

Aprovado em novembro de 2018.

\begin{abstract}
RESUMO
Com a expansão do conceito de cidades desenvolvidas sustentavelmente, os Sistemas de Mapeamento Móveis (SMM) surgem como ferramenta útil, que através do levantamento fotogramétrico terrestre permite a coleta de um grande volume de informações georreferenciadas para atividades que exigem tomadas de decisões. Outra ferramenta que permite visualização de informação geográfica e que atualmente vem ganhando destaque são as plataformas conhecidas como WebSIG. O termo WebSIG refere-se a aplicações que distribui dados espaciais para os usuários através de um navegador Web, assim as aplicações WebSIG disponibilizadas pelo Municípios permitem aos usuários o acesso a informação geográfica variada e de uma forma intuitiva, usando apenas o seu browser de internet, sem necessidade de instalação de qualquer software. Com esse intuito, esse trabalho tem por objetivo apresentar o Sistema de Mapeamento Móvel e um WebSIG, ambos desenvolvido na Universidade Federal de Uberlândia (UFU) para visualização de mapas digitais, imagens aéreas, imagens de satélite e imagens de fachadas de imóveis com rapidez e segurança, úteis para uma gama de usuários que podem visualizar, consultar e analisar dados geográficos do município.
\end{abstract}

PALAVRAS-CHAVE: WebSIG. Sistema de Mapeamento Móvel. Fotogrametria Digital.

\begin{abstract}
With the expansion of the concept of sustainably developed cities, Mobile Mapping Systems (SMM) emerge as a useful tool, which through the terrestrial photogrammetric survey allows the collection of a large volume
\end{abstract}

${ }^{1}$ Instituto de Geografia, Universidade Federal de Uberlândia, Brasil. E-mail: rodrigogallis@ufu.br; rluisbarbosa@ufu.br; samuelrodriguescrs@gmail.com; marcelo.af@ufu.br

2 Integral Soluções Analíticas, Brasil. E-mail: integralsol@integralsol.com.br 
of georeferenced information for activities that require decision-making. Another tool that allows visualization of geographic information and that is currently gaining prominence are the platforms known as WebSIG. The term WebSIG refers to applications that distribute spatial data to users through a WebBrowser, so the WebSIG applications made available by the Municipalities allow users to access varied geographic information in an intuitive way using only their Internet browser without the installation of any software. The purpose of this paper is to present the Mobile Mapping System and a WebSIG, both developed at the Federal University of Uberlandia (UFU) for the visualization of digital maps, aerial images, satellite images and facade images quickly and easily, useful for a range of users who can view, query and analyze geographical data of the municipality.

KEYWORDS: WebSIG. Mobile Mapping System. Digital Photogrammetry.

$$
* * *
$$

\section{Introdução}

A integração da Cartografia com tecnologias computacionais proporcionou o desenvolvimento dos Sistemas de Informação Geográfica (SIG's), que são ferramentas gerenciais para organizar, processar e administrar grandes volumes de informações geográficas para atividades que exigem tomadas de decisões. Tais sistemas necessitam de atualizações periódicas e por isso deve haver um grande dinamismo na atualização destes dados; desse modo também há a necessidade de técnicas confiáveis para realização das coletas de informações.

Não é novidade dizer que o Brasil ainda é um país de carências, e que as dimensões continentais do país ainda não foram adequadamente mapeadas, verificando-se grandes porções do território sem cobertura cartográfica. É comum também observar que as cartas topográficas de regiões já mapeadas podem estar desatualizadas. Devido a esta crescente necessidade de atualização de dados, houve um grande avanço nas pesquisas, buscando novas técnicas de coleta de informações espaciais e melhoramento das técnicas já existentes (OLIVEIRA, 2003).

Uma delas são os Sistemas Móveis de Mapeamento (SMM), que vem se destacando principalmente devido à sua eficiência e economia. Estes sistemas 
proporcionam sequências de imagens, que permitem a análise e medição de pontos para fins de levantamento e mapeamento fotogramétrico ou simples observação para avaliação das condições das vias de transporte e de seu entorno. Essas condições são físicas, geométricas e ambientais, como, por exemplo, as condições do pavimento, largura, rampas, acostamentos ou calçadas, arborização, sinalização horizontal e vertical, segurança para pedestres e condutores.

Os Sistemas Móveis de Mapeamento representam uma metodologia de levantamento de dados que integra sensores de posicionamento e imageamento, permitindo o mapeamento pormenorizado do mundo real. Com a expansão do conceito de cidades desenvolvidas sustentavelmente, o SMM surge como ferramenta ideal através do levantamento fotogramétrico terrestre para dar o suporte necessário para uma visão completa do setor de vias e todos seus componentes, o que traz consigo variedades de aplicações para gestores públicos no controle, monitoramento e melhoramento de bases cartográficas (GALLIS, 2002). O potencial de informações que podem ser abstraídas da análise dos dados coletados em campo é útil para o monitoramento de áreas que estão sujeitas as constantes intervenções humanas e nesse sentido a concepção de um sistema de coleta de dados georreferenciados e de um sistema de gerenciamento integrado dessas informações torna-se essencial.

Outra ferramenta SIG que permite visualização de informação geográfica e que atualmente vem ganhando destaque são as plataformas conhecidas como WebSIG. O termo WebSIG refere-se a aplicações que distribui dados espaciais para os usuários através de um navegador Web, assim as aplicações WebSIG disponibilizadas pelos Municípios permitem aos usuários o acesso a informação geográfica variada e de uma forma intuitiva, usando apenas o seu browser de internet, sem necessidade de instalação de qualquer software. Esta combinação permite o acesso a informações espaciais, assim como uma grande variedade de ferramentas de análise e serviços de 
dados usando o navegador da web (PENG e TSOU, 2003), alcançando um maior número de usuários (TANG e SELWOOD, 2003).

Com esse intuito, esse trabalho tem por objetivo apresentar o Sistema de Mapeamento Móvel Terrestre de baixo custo e mostrar uma prova de conceito que coletou imagens da arborização urbana e imagens das fachadas dos imóveis na área urbana de Monte Carmelo-MG, e apresentar o WebSIG, desenvolvido para a disponibilização dos mapas digitais, imagens aéreas, imagens de satélite, coordenadas e imagens das árvores, imagens de fachadas dos imóveis com rapidez e segurança, para uma gama de usuários que podem visualizar, consultar e analisar dados geográficos do município remotamente através de um navegador Web utilizando-se de funcionalidade SIG básicas, facilitando assim o acesso a informação e a dados espaciais para suporte a uma série de decisões e de visualização de dados espaciais voltados para o ambiente Web.

Os dados cadastrados no WebSIG podem ser utilizados num futuro para estudos da dinâmica urbana, planejamento urbano, quantificação das espécies de arvores existentes na mancha urbana do município, cadastro técnico multifinalitário, classificações dos tipos de construções de acordo com a finalidade e atualização da Planta Genérica de Valores da cidade.

\section{$2 \quad$ Material e Métodos}

\subsection{Sistema de Mapeamento Móvel Terrestre}

O sistema de mapeamento móvel foi idealizado e construído com materiais adquiridos com recursos CNPq, Projeto Universal 483413/20127: 4 câmaras GoPro Black Hero 3.0, 4 cartões de memória de 64GB, 1 sensor GNSS/INS Xsens. Além desses equipamentos, foram utilizados um Notebook i7/1Tb, um automóvel e um suporte metálico para fixação das câmaras e do sensor GNSS/INS, acoplado a ventosas para colocação no teto do automóvel. 
O SMM foi utilizado para coleta de informações georreferenciadas de ruas, capturando imagens e vídeos digitais continuamente, permitindo a posterior obtenção da coordenada tridimensional das feições levantadas.

O planejamento foi realizado em um sistema implementado em C\# e a biblioteca DotSpatial, que utiliza uma base em formato shapefile feita com a vetorização das quadras através do Google Maps. Nesse sistema de planejamento, o operador informa o sentido de cada rota (mão única, mão dupla) e o início e fim de cada via, aqui chamado de percurso. Esses percursos formam um grafo orientado e um algoritmo de caminho mínimo informa qual percurso é o próximo a ser levantado. O sentido do trânsito das vias é obtido com do Google Maps e se necessário, durante o levantamento, é possível atualizar o sentido da via. Esses dados ficam em um banco de dados local em SQL Server.

Após o planejamento e com o sistema embarcado no automóvel, a posição é atualizada pelo GNSS e é iniciado o processo de coleta (vídeos e as coordenadas da posição e orientação). As câmaras são ligadas ao mesmo tempo com um controle remoto, via wifi. Ao começar um percurso, as câmaras são ligadas e o vídeo é capturado, bem como o sinal GNSS/INS. Ao finalizar o percurso, o operador encerra a rota e o sistema indica qual a próxima rota que é a melhor para otimizar o tempo, e o processo se repete, até finalizar todas as rotas planejadas.

Para o levantamento foram utilizadas câmaras na horizontal, com resolução de vídeo de 4K, uma para cada lado da via e duas câmaras rotacionadas $90^{\circ}$, com resolução de vídeo de 1080p (Figuras 1 e 2). As câmaras rotacionadas são usadas para visualizar edificações com mais de um andar, árvores e ativos da iluminação pública.

Figura 1 - Configuração das câmaras Figura 2 - Sistema de Mapeamento utilizado 


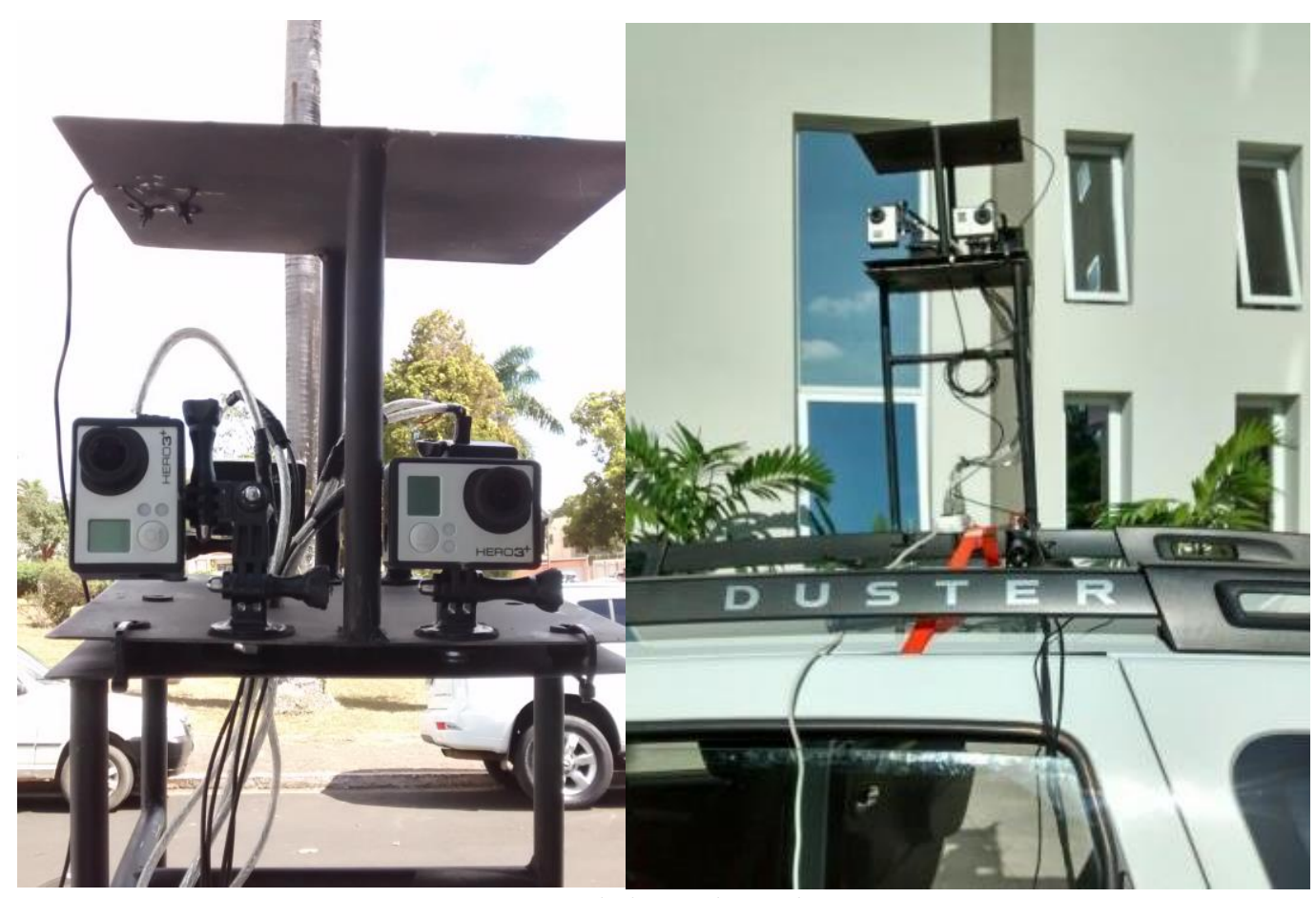

Fonte: elaborada pelos autores.

Ao fim do levantamento, os vídeos são descarregados dos cartões de memória das câmaras para o Notebook e feita uma cópia em um HD externo. Verificada a integridade dos vídeos, os mesmos são apagados do cartão de memória para o próximo levantamento.

Com o encerramento do levantamento, o próximo passo é georreferenciar os vídeos, para que, em um aplicativo, chamado de recorte de fachadas, o usuário assiste ao vídeo e ao verificar que a feição a ser estudada (fachada, árvore, iluminação pública) está centralizada, faz o recorte, ou seja, é como se uma foto fosse feita e a informação vai para o banco de dados.

A utilidade desse sistema se mostra ampla, com uma gama de aplicações na engenharia e serviços de utilidade pública utilizando-se da integração entre sistemas de imageamento digital e de navegação (posicionamento global e inercial).

Entre outras características pode-se destacar também:

- capacidade de determinar coordenadas tridimensionais de feições com base em imagens em posicionamento global, sem a necessidade de 
pontos de controle espacial no terreno, apenas com processo de fototriangulação;

- rapidez e agilidade na coleta de informações em campo, propiciando uma considerável redução dos custos com tempo de levantamento;

- bom desempenho em áreas em que não haja obstrução do sinal GNSS devido aos obstáculos (árvores, viadutos, grandes construções etc.);

Em alguns sistemas operantes no mundo as câmaras obtêm as imagens continuamente, sensores laser fazem varredura gerando nuvens de pontos que podem ser utilizadas para geração de maquetes tridimensionais, os receptores GPS dão o posicionamento global, a IMU (Inertial Measurement Unit) mede a orientação angular do sensor de imagem e também complementa as posições quando os dados GPS estão prejudicados por causa das interferências das estruturas ao longo das ruas e rodovias, conforme já mencionado. Na Tabela 1, encontram-se alguns sistemas operantes no mundo e uma breve descrição dos equipamentos e sensores embarcados nos mesmos (TAO, 2000; TOSCHI et al., 2015).

Tabela 1 - Tabela de alguns sistemas de mapeamento existentes (dos precursores que utilizavam sensores de imageamento como câmaras VHS até os atuais com laser scanner e câmaras panorâmicas $360^{\circ}$ embarcadas)

Sensores

\begin{tabular}{|c|c|c|c|c|}
\hline Nome/referência & Desenvolvimento & Plataforma & Navegação & Mapeamento \\
\hline $\begin{array}{l}\text { GPSVanTM } \\
\text { Goad, 1991; } \\
\text { Novak, } 1991\end{array}$ & $\begin{array}{c}\text { The Ohio State } \\
\text { University }\end{array}$ & Van/trem & $\begin{array}{c}\text { GPS, } 2 \\
\text { giroscópios, } 2 \\
\text { odomêtros } \\
\text { (contador de } \\
\text { velocidade) }\end{array}$ & $\begin{array}{c}2 \text { câmaras } \\
\text { monocromáticas } \\
\text { digitais CCD, } 2 \\
\text { câmaras VHS } \\
\text { coloridas }\end{array}$ \\
\hline $\begin{array}{c}\text { CDSS } \\
\text { Benning and } \\
\text { Aussems, } 1998\end{array}$ & $\begin{array}{c}\text { Geodetic Institute } \\
\text { Aachen }\end{array}$ & Van & $\begin{array}{c}\text { GPS, } 2 \\
\text { odomêtros, } \\
\text { baromêtros }\end{array}$ & $\begin{array}{c}2 \text { câmaras } \\
\text { monocromáticas } \\
\text { digitais CCD }\end{array}$ \\
\hline $\begin{array}{c}\text { KiSSTM } \\
\text { Hock et al., 1995; } \\
\text { Sternberg et al., } \\
2001\end{array}$ & $\begin{array}{l}\text { University of the } \\
\text { Federal } \\
\text { Armed Forces } \\
\text { Munich }\end{array}$ & Van & $\begin{array}{c}\text { GPS, IMU (2 } \\
\text { giroscópios, } 3 \\
\text { acceleromêtros) } \\
\text { odomêtro, } \\
\text { baromêtro }\end{array}$ & $\begin{array}{c}2 \text { câmaras } \\
\text { monocromáticas } \\
\text { digitais CCD, } 2 \\
\text { câmaras VHS } \\
\text { coloridas }\end{array}$ \\
\hline Nome/referência & Desenvolvimento & Plataforma & Navegação & Mapeamento \\
\hline VISATTM & & Van & & $\begin{array}{c}8 \text { câmaras } \\
\text { monocromáticas }\end{array}$ \\
\hline
\end{tabular}

Rev. Bras. de Cartografia, vol. 70, Edição Especial "XXVII Congresso Brasileiro de 


\begin{tabular}{|c|c|c|c|c|}
\hline $\begin{array}{c}\text { Schwarz et al., } \\
1993 ; \\
\text { El-Sheimy and } \\
\text { Schwarz, } 1999\end{array}$ & $\begin{array}{l}\text { University of } \\
\text { Calgary }\end{array}$ & & $\begin{array}{l}\text { GPS dupla } \\
\text { frequência, } \\
\text { IMU }\end{array}$ & $\begin{array}{l}\text { digitais CCD, } 1 \\
\text { câmara VHS } \\
\text { colorida }\end{array}$ \\
\hline $\begin{array}{c}\text { TruckMAPTM } \\
\text { Reed et al., } 1996\end{array}$ & $\begin{array}{l}\text { John E. Chance } \\
\text { and Associates }\end{array}$ & Van & $\begin{array}{l}\text { GPS, sensores } \\
\text { de atitude } \\
\text { digitais }\end{array}$ & laser range finder \\
\hline $\begin{array}{c}\text { GIMTM } \\
\text { Coetsee et al., } \\
1994\end{array}$ & NAVSYS Corp. & Caminhão & $\begin{array}{l}\text { GPS, IMU de } \\
\text { baixo custo }\end{array}$ & $\begin{array}{c}1 \text { câmara digital } \\
\text { CCD, } 1 \text { câmara VHS }\end{array}$ \\
\hline $\begin{array}{c}\text { WUMMS } \\
\text { Li et al., } 1999\end{array}$ & $\begin{array}{c}\text { Wuhan Technical } \\
\text { University }\end{array}$ & Caminhão & $\begin{array}{c}\text { GPS, sensor } \\
\text { deadreckoning } \\
\text { não } \\
\text { especificado }\end{array}$ & $\begin{array}{l}1 \text { câmara digital } \\
\text { CCD, laser range- } \\
\text { finder }\end{array}$ \\
\hline $\begin{array}{l}\text { GPSVisionTM } \\
\text { Lambda Tech., } \\
2002\end{array}$ & Lambda Tech. & Van & GPS, IMU & $\begin{array}{l}2 \text { câmaras digitais } \\
\text { CCD coloridas }\end{array}$ \\
\hline $\begin{array}{c}\text { ON-SIGHTTM } \\
\text { Transmap, } 2002\end{array}$ & Transmap Corp. & Van & GPS, IMU & $\begin{array}{l}5 \text { câmaras digitais } \\
\text { CCD }\end{array}$ \\
\hline $\begin{array}{c}\text { MoSES } \\
\text { Graefe et al., } 2001\end{array}$ & $\begin{array}{l}\text { University of the } \\
\text { Federal } \\
\text { Armed Forces } \\
\text { Munich }\end{array}$ & Van & $\begin{array}{c}\text { GPS, IMU, } \\
\text { odomêtro } \\
\text { baromêtro }\end{array}$ & $\begin{array}{c}2 \text { câmaras digitais } \\
\text { CCD coloridas } \\
\text { (possível laser } \\
\text { scanner e câmara de } \\
\text { vídeo digital } \\
\text { colorida) }\end{array}$ \\
\hline $\begin{array}{c}\text { GEOVan } \\
\text { Geospan, } 2002\end{array}$ & Geospan Corp. & Van & $\begin{array}{l}\text { GPS, dead } \\
\text { reckoning }\end{array}$ & 10 câmaras \\
\hline $\begin{array}{c}\text { RGIAS } \\
\text { Rowe, } 2002\end{array}$ & $\begin{array}{l}\text { Rowe Surveying } \\
\text { and Engineering } \\
\text { Co. } \\
\end{array}$ & Caminhão & GPS & Laser \\
\hline $\begin{array}{c}\text { MANDLI } \\
\text { Mandli, } 2002\end{array}$ & Mandli Comm. & Van & GPS & $\begin{array}{l}2 \text { câmaras digitais } \\
\text { CCD }\end{array}$ \\
\hline $\begin{array}{c}\text { UMMD } \\
\text { Silva et al., } 2000\end{array}$ & $\begin{array}{c}\text { Universidade } \\
\text { Estadual Paulista }\end{array}$ & Van & GPS & $\begin{array}{l}2 \text { câmaras de vídeo } \\
\text { digital DVCAM }\end{array}$ \\
\hline GeoMobil & $\begin{array}{c}\text { Institut Cartogràfic } \\
\text { de Catalunya }\end{array}$ & Van & GPS, IMU & $\begin{array}{c}\text { Laser scanner } \\
\text { terrestre, } 2 \text { câmaras } \\
\text { digitais CD }\end{array}$ \\
\hline IP-S3 & TOPCON & Carro & GPS, IMU & $\begin{array}{l}1 \text { scanner com } \\
\text { alcance de } 100 \text { m e } \\
\text { câmera esférica com } \\
8000 \text { x } 4000 \text { pixel }\end{array}$ \\
\hline MX8 & TRIMBLE & Carro & GPS, IMU & $\begin{array}{l}\text { Laser Scanner VQ- } \\
250 \text { com } 500 \mathrm{~m} \text { de } \\
\text { alcance ou } 1-2 \mathrm{VQ} \text { - } \\
450 \text { com } 800 \mathrm{~m} \text { de } \\
\text { alcance e } 7 \text { câmeras } \\
\text { de } 5 \text { megapixel }\end{array}$ \\
\hline Nome/referência & Desenvolvimento & Plataforma & Navegação & Mapeamento \\
\hline Street Mapper & 3D Laser Mapping & Carro & GPS, IMU & $\begin{array}{c}\text { Laser Scanner } \\
\text { VUX-1HAe câmara }\end{array}$ \\
\hline
\end{tabular}

Rev. Bras. de Cartografia, vol. 70, Edição Especial "XXVII Congresso Brasileiro de 


\begin{tabular}{c|c|c|c|c}
\hline & & & & $\begin{array}{c}\text { panorâmica de 12 } \\
\text { megapixel }\end{array}$ \\
\hline VMX-250 & RIEGL & Carro & GPS, IMU & $\begin{array}{c}\text { 2 canhões Laser } \\
\text { Scanner VQ-250 e 6 } \\
\text { câmaras 5 megapixel }\end{array}$ \\
\hline Dynascan S250 & Renishaw & Carro & GPS, IMU & 2 scanner(s) \\
\hline Lynx SG1 & TELEDYNE & Carro & GPS, IMU & $\begin{array}{c}2 \text { scanners e 5 } \\
\text { câmaras, 5 } \\
\text { megapixel }\end{array}$ \\
\hline Lynx MG1 & OPTECH & Carro & GPS, IMU & $\begin{array}{c}1 \text { scanner e uma } \\
\text { câmara panorâmica }\end{array}$ \\
\hline MMS-X & MITSUBISHI & Carro & GPS, IMU & $\begin{array}{c}\text { laser scanners e 6 } \\
\text { câmaras de 5 } \\
\text { megapixel }\end{array}$ \\
\hline \multirow{2}{*}{ Leica Geosystems } & Leica Pegasus & Carro & GPS, IMU & $\begin{array}{c}\text { Laser scanner ZF } \\
9012 \text { e cameras 2000 } \\
\text { x 2000 pixel }\end{array}$ \\
\hline
\end{tabular}

Fonte: elaborada pelos autores.

No levantamento realizado com o sistema de Mapeamento Móvel desenvolvido na UFU a taxa de captura utilizada no sensor GNSS/INS Xsens foi de uma coordenada a cada 1 segundo. Juntamente com as coordenadas, o arquivo também salvou os ângulos de orientação exterior (roll, pitch, yaw), conforme Figura 3. Clicando-se em cada ponto que corresponde a uma coordenada capturada é possível visualizar as informações de posição e orientação exterior fornecida pelo equipamento.

Figura 3 - Exemplo de arquivo de saída do GNSS/INS Xsens visualizado no Google Maps.

Rev. Bras. de Cartografia, vol. 70, Edição Especial "XXVII Congresso Brasileiro de 


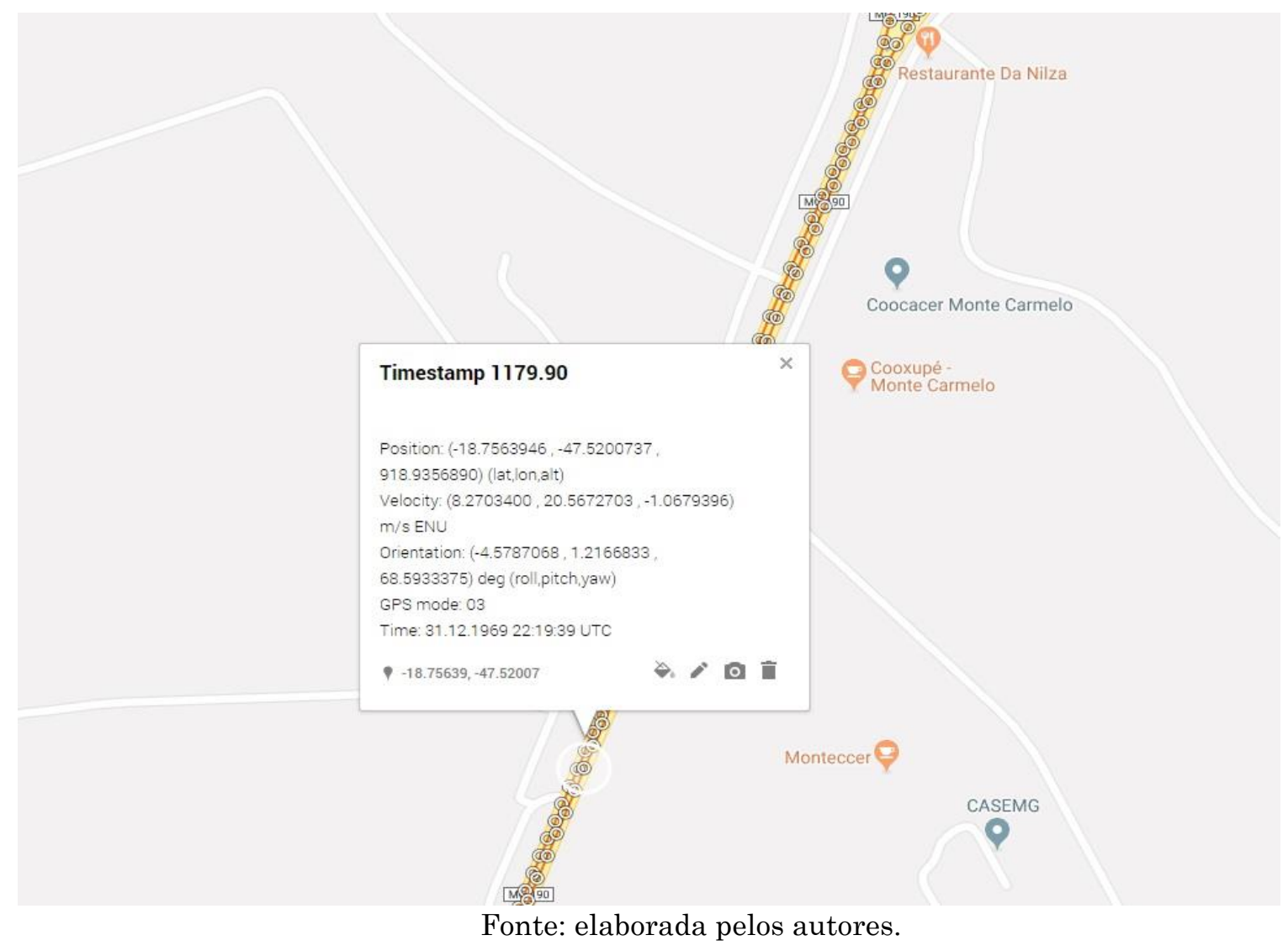

\subsection{WebSIG}

O desenvolvimento da Internet nas últimas décadas mostrou-se um fator determinante para a evolução dos Sistemas de Informações Geográficas (SIG), permitindo o compartilhamento de informações espaciais diretamente e descentralizando serviços de dados espaciais, estando estes disponíveis através da Internet, com a integração de tecnologias SIG com a Web criando o conceito de WebSIG (YANG, WONG, YANG, KAFATOS e LI, 2004). O sistema WebSIG é um sistema de informações geográficas online, hospedado em servidor web. Através de um navegador de internet, é possível consultar e manipular informações georreferenciadas de acordo com o interesse do usuário.

Diante do crescente volume de informações geográficas que as empresas privadas e agências governamentais utilizam, e as demandas específicas de análise espacial, o sistema WebSIG surgiu como ferramenta, 
servindo como suporte interativo para visualização e gerenciamento de dados espaciais gerados pelo uso do sistema de mapeamento móvel terrestre utilizado (Figuras 4 e 5).

Figura 4 - WebSIG do Laboratório de Sensoriamento Remoto e Fotogrametria do curso de Engenharia de Agrimensura e Cartográfica da Universidade Federal de Uberlândia, criado para visualização dos levantamentos realizados com o Sistema de Mapeamento Móvel.

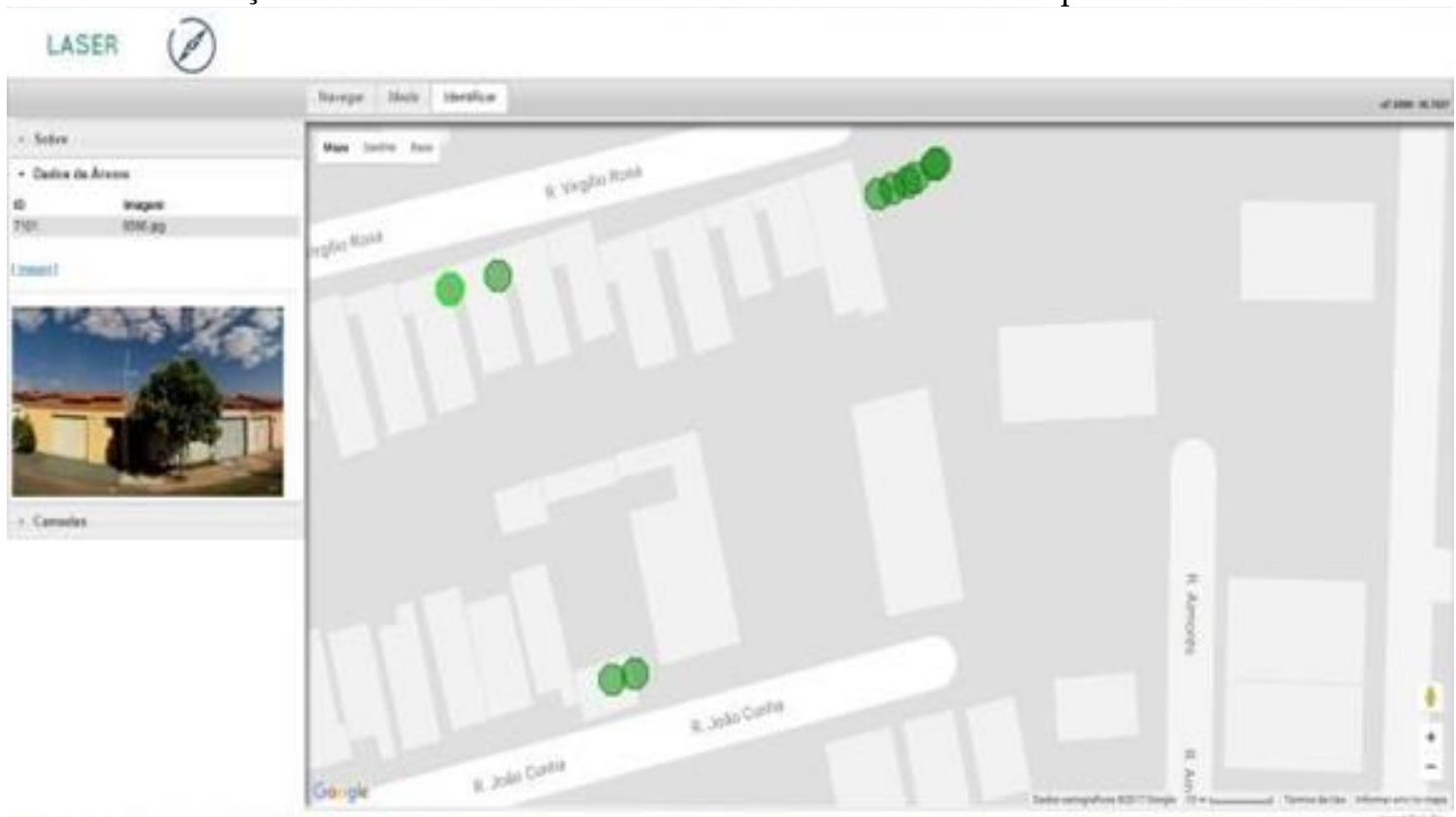

Fonte: elaborada pelos autores. 
Figura 5 - Pontos que representam as árvores e que foram extraídas através das imagens coletadas com o Sistema de Mapeamento Móvel

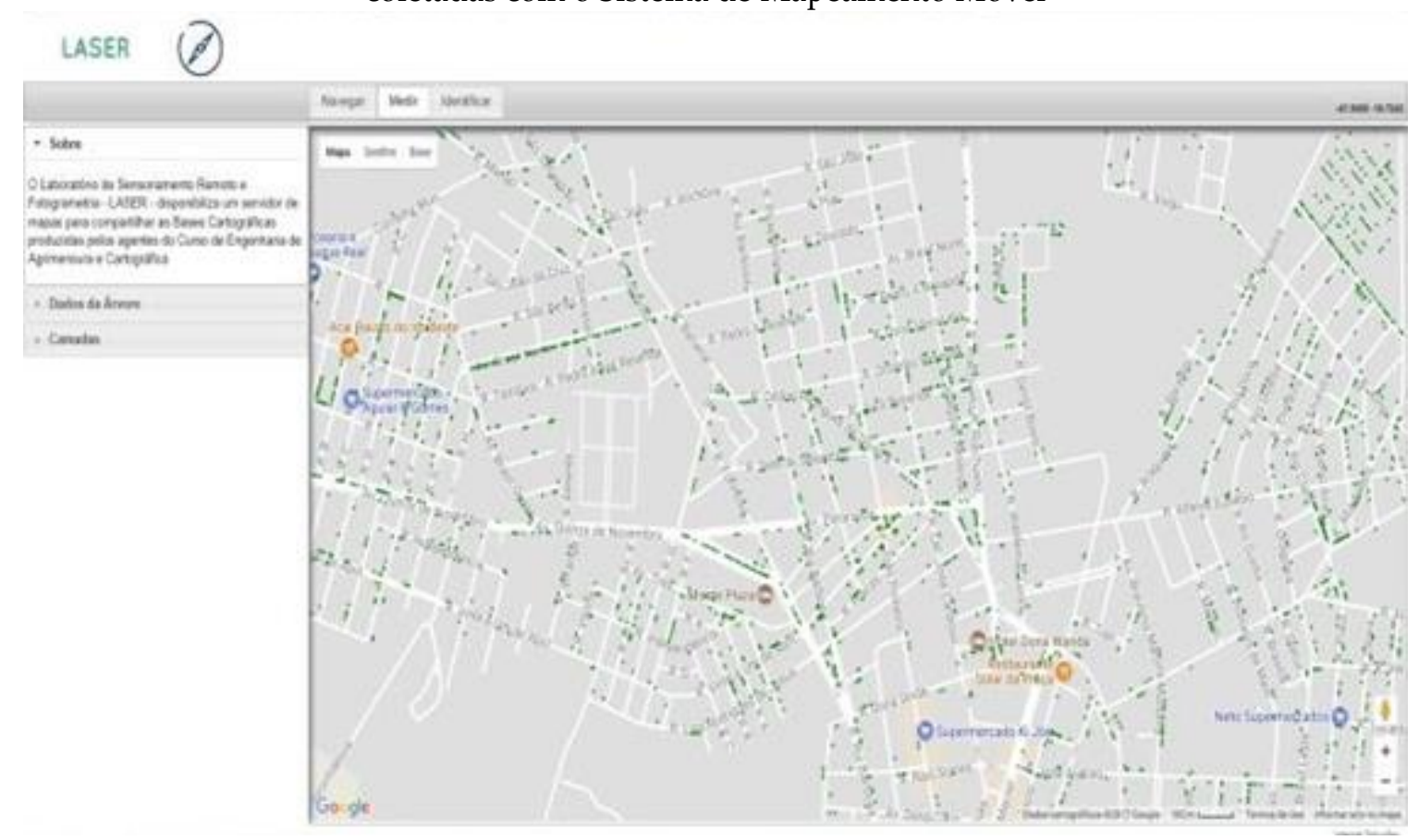

Fonte: elaborada pelos autores.

Os aplicativos WebSIG fornecem várias funções e funcionalidades aos usuários, como se fosse um software SIG desktop clássico. Permitem a visualização básica (panorâmica e zoom), seleção de camadas de dados e visualização das informações associadas às diferentes entidades espaciais, às ferramentas de maior complexidade, como medir distâncias e áreas ou realizar análises espaciais que podem exigir um nível mais alto de processamento de dados (DRUMMOND e FRENCH, 2008).

Os WebSIG, permitem sua utilização através de navegadores Web, com funcionalidades próprias dos SIG proporcionando uma plataforma de acesso à informação geográfica independentemente dos sistemas operacionais.

\section{Resultados e Discussões}


Toda a mancha urbana da cidade de Monte Carmelo-MG foi levantada em dois dias de campo (cerca de 16 horas). A taxa de captura dos dados GNSS/INS foi de $1 \mathrm{~Hz}$, e além da posição (latitude, longitude), foram armazenados dados de orientação do sensor (roll, pitch, yaw), o tempo universal e a altitude. Os dados foram processados por software proprietário e exportados para o formato kmz, para visualização no Google Earth e formato .txt, para conversão em formato shapefile. Os dados brutos ocuparam aproximadamente $12 \mathrm{MB}$ de memória.

É feita uma limpeza nos dados GNSS/INS, pois o sensor começa a capturar dados desde o início do levantamento, o que gera uma grande quantidade de dados redundantes. Como a velocidade do carro é de cerca de $30 \mathrm{~km} / \mathrm{h}$, enquanto captura os vídeos de cada percurso, isso gera um dado GNSS/INS a cada $8 \mathrm{~m}$, aproximadamente. O processo de limpeza é realizado com o georreferenciamento e a sincronização dos vídeos, pois somente onde tem vídeo gravado, os dados GNSS/INS são armazenados e depois carregados em um banco dados SQL Server, para ser utilizado no software de recorte das imagens de fachadas, de árvores e outras feições.

Foram percorridas 328 rotas, o que produziu 1312 arquivos de vídeo, com um espaço de 699GB. O total deslocado foi de 297,2km. Através do uso do sistema de mapeamento móvel, foi possível o levantamento fotogramétrico de todas as árvores existentes na mancha urbana do município. As coordenadas georreferenciadas extraídas das 7336 árvores do município estão representadas no WebSIG, Figura 5. Na Figura 6 exemplo de uma imagem de árvore e ativo de iluminação pública.

Figura 6 - Exemplo de imagem de árvore e ativo de iluminação pública coletada pelo SMM desenvolvido 


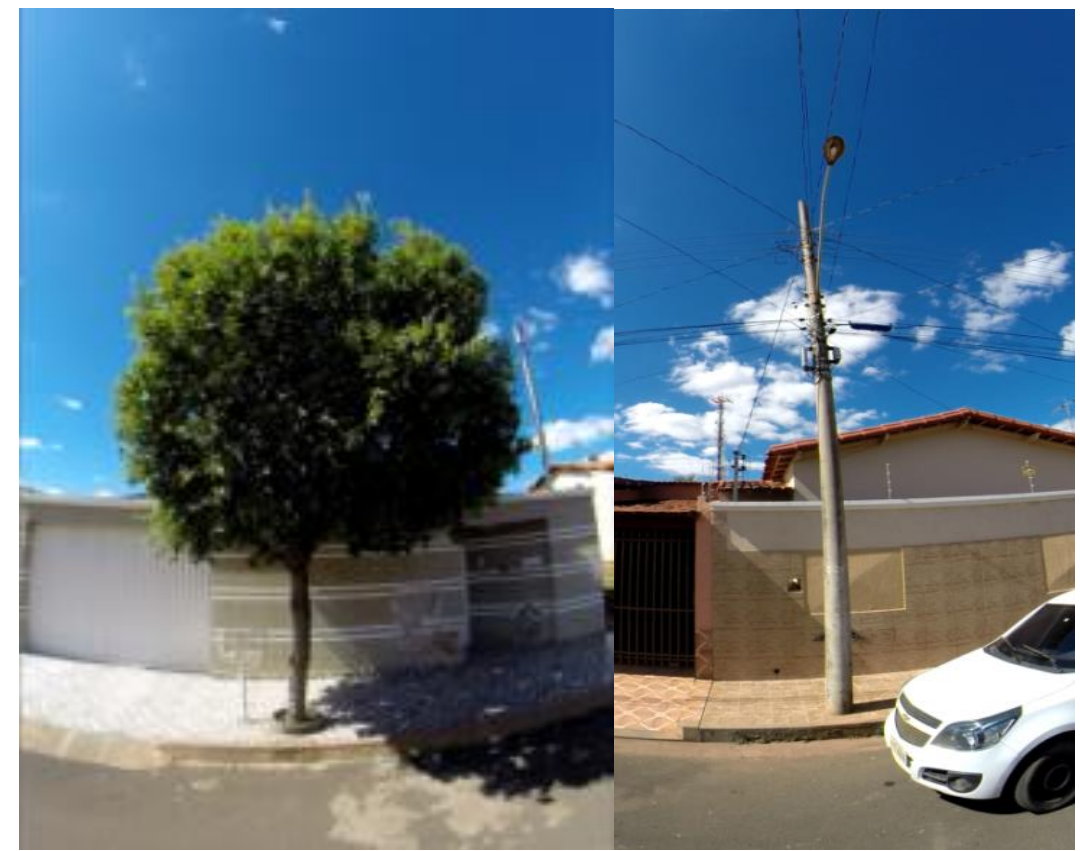

Fonte: elaborada pelos autores.

A qualidade do vídeo 4k gera uma imagem de alta resolução, que permite inclusive enxergar a numeração do lote e com um sistema de reconhecimento de números (SILVA et al., 2013) o cadastro desse dado pode ser realizado de modo automático. Com os vídeos de $1080 \mathrm{p}$, o processo de recorte é o mesmo e é possível observar os ativos da iluminação púbica e as árvores. Na Figura 7, exemplo de um recorte de imagem de fachada para fins de visualização.

Figura 7 - Exemplo de uma fachada recortada do vídeo 4k 


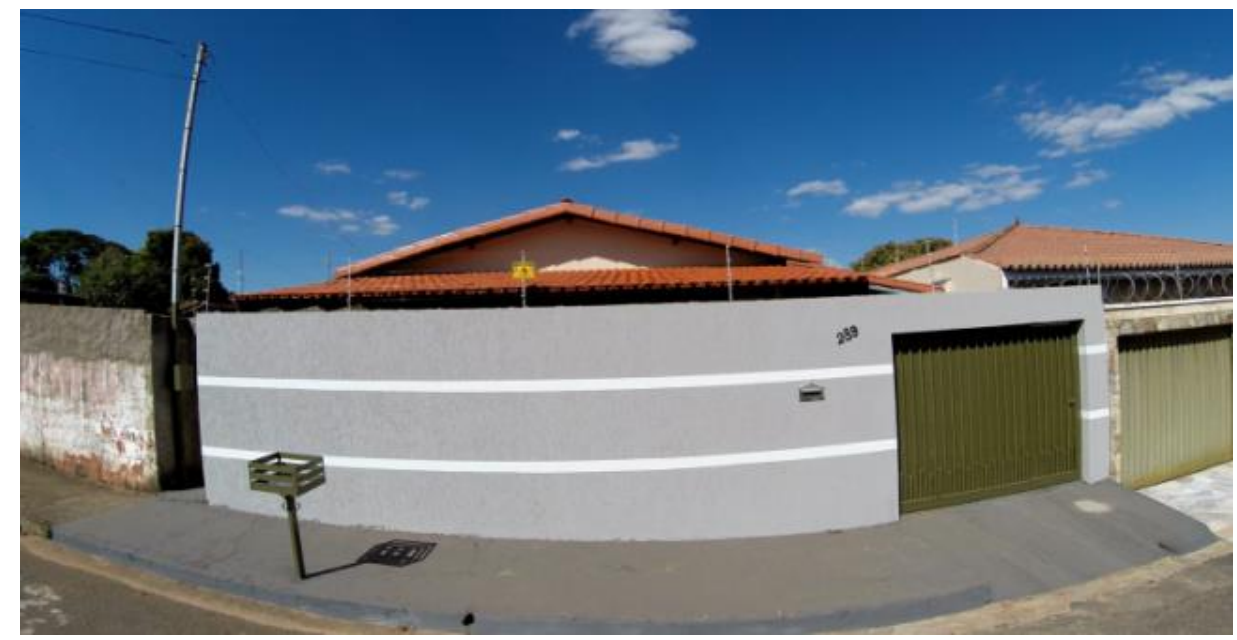

Fonte: elaborada pelos autores.

O conjunto de vídeos e fotos frontais integrados ao WEBSIG torna-se uma importante ferramenta para o cadastro imobiliário. Dentre as informações que podem ser extraídas dos dados levantados, destacam-se: o levantamento das edificações que se encontram em construção, o padrão de construção (classificação) dos imóveis, conforme visualização externa, o uso dos imóveis - classificação mobiliária (residencial, comercial, industrial), a presença ou não de guias e calçadas, o tipo de pavimentação, o tipo de construção (térrea, sobrado, edifício etc.), posicionamento dos postes e arborização, identificação das placas de trânsito etc.

\subsection{Funcionalidades do WebSIG desenvolvido}

Dentre as funcionalidades do sistema desenvolvido, podemos destacar:

- "Hipertlink" entre a imagem e a feição representada no mapa digital.

- Medição de distâncias em linha reta, e cálculo de áreas de polígonos selecionáveis.

- Identificação de feições selecionadas. Essa função mostra características e pontos importantes sobre a variável desejada, tais 
como classificação, fotografia, número de identificação e coordenadas georreferenciadas.

- Geoespacialização de todas as áreas levantadas (dados georreferenciados).

- Disponibilidade de "full time" do WebSIG, fato resultante de todo o conteúdo estar embasado na web.

- A possibilidade de consulta pública de maneira direta e facilitada, o que traz perspectivas de uma base eficiente para a utilização em órgãos e entidades municipais.

- Mapeamento corporativo, que traz a vantagem de colaboração e auxílio em pesquisas científicas.

\section{Conclusão}

Considerando o crescente uso da tecnologia WebSIG e a consequente necessidade de dados atualizados, pode-se concluir que a importância de um meio eficaz e rápido de coleta de informações georreferenciadas será cada vez mais solicitado para fornecer dados georreferenciados para estas novas tecnologias de visualização e gerenciamento de dados geográficos online. Considerando-se ainda a possibilidade de utilização de sensores digitais em levantamentos realizados utilizando a metodologia existente no mapeamento móvel, especialmente câmaras digitais, faz com que o sistema desenvolvido, com características de resposta rápida e baixo custo, atenda às administrações públicas e privadas voltadas aos serviços de Cartografia, no que se refere a:

- áreas de mapeamento temático, topográfico e cadastral;

- atualizações cartográficas;

- projetos em levantamentos de feições urbanas (monitoramento urbano);

A ideia é que o WebSIG desenvolvido ofereça um ambiente para visualização de todos os produtos de mapeamento gerados pelas pesquisas 
realizadas no Curso de Engenharia de Agrimensura e Cartográfica da Universidade Federal de Uberlândia. A intenção é utiliza-lo para representar os mapas gerados pelos dados coletados com sistema de mapeamento móvel assim como integração entre a feição e sua correspondente imagem.

A possibilidade de utilização de sensores digitais em levantamentos realizados utilizando a metodologia existente no mapeamento móvel, especialmente câmaras digitais, faz com que o sistema desenvolvido, com características de resposta rápida e baixo custo, atenda às administrações públicas e privadas voltadas aos serviços de Cartografia, no que se refere a: áreas de mapeamento temático, topográfico e cadastral, atualizações cartográficas, projetos em levantamentos de feições urbanas (monitoramento urbano) e levantamento de rodovias e ferrovias.

Com o sistema desenvolvido, o custo para um levantamento de pequenas cidades, onde não existe um voo aerofotogramétrico, pode ser uma alternativa viável, para os gestores terem a disposição uma base cartográfica e um sistema para um processo de atualização cadastral. Somente com uma simples inspeção de lotes onde residências foram construídas ou reformadas, os gestores podem melhorar a fiscalização além de obterem dados para fins de atualização da planta genérica de valores. Também é possível com esses dados, implementar rotinas de consultas para a contagem e georeferenciamento de serviços públicos, estabelecimentos comerciais e de serviços, além de verificar a qualidade do arruamento, acessibilidade e demais requisitos da legislação.

Os próximos passos desse trabalho, será implementar o sistema para fazer o recorte das fachadas e ter cada fachada georreferenciada, utilizandose técnicas fotogramétricas. Também será realizado um inventário das árvores do município que estão no passeio e aparecem nos vídeos. Com a qualidade do vídeo, é possível, para especialistas identificarem as espécies.

Os ativos de iluminação pública também farão parte do banco de dados e de imagens que serão visualizados no WebSIG e os dados de altitude do sensor GNSS/INS serão utilizados para a geração do modelo digital do terreno 
do município. Cabe destacar que os dados desse trabalho estão sendo compartilhados com os demais pesquisadores interessados e também com a prefeitura da cidade de Monte Carmelo.

\section{Agradecimentos}

Os autores agradecem ao CNPq pelo apoio através do Edital Universal Processo CNPq 483413/2012-7 e a CAPES pela concessão de Bolsa de Estágio Sênior no Exterior (Processo 99999.002696/2015-00).

\section{Referências}

CUNHA, S. R., BARBOSA, R. L., HIRAGA, A. K., GALLIS, R. B. A., FARIA, M. A. WEBSIG alimentado com dados coletados com Sistema de Mapeamento Móvel Terrestre. Anais do XXVII Congresso Brasileiro de Cartografia e XXVI Exposicarta, 2017, SBC, Rio de Janeiro - RJ, pp. 735-738.

DRUMMOND, W. J.; FRENCH, S. P. The Future of GIS in Planning. Journal of the American Planning Association. nº 74, 2008. pp. 161-174

GALLIS, R. B. A.; OLIVEIRA, R. A.; SILVA, J. F. C. Aplicação da unidade móvel de mapeamento digital para construir um banco de imagens georreferenciadas. Revista de Iniciação Científica. v.2, 2000. pp. 212 - 221.

GALLIS, R. B. A.; SILVA, J. F. C.; CAMARGO, P.O.; BARBOSA, R. L. Mapeamento móvel no Brasil: Resultados obtidos com a utilização da Unidade Móvel de Mapeamento Digital In: Série em Ciências Geodésicas Ed. Curitiba : Imprensa Universitária, v.2, 2002. pp. 248-266.

HABIB A. F.; UEBBING, R. \& NOVAK, K. Automatic Extraction of Road Signs from Terrestrial Color Imagery. Photogram. Eng. \& Remote Sens. (Journal. of the Amer. Soc. of Photogram. \& Remote Sens.) 65(7). 1999. pp 597-602.

LI, R.; CHAPMAN, A.; QIAN, L.; XIN, Y.; TAO, C. VISAT: a real time system for highway spatial information acquisition. In: ASPRS-ACSM Annual Convention and Exposition, Reno. Proceedings... Bethesda: ASPRS \& ACSM, v.1, 1994, pp.344-9. 
OLIVEIRA, R. A. Concepção, Desenvolvimento e Aplicação do Banco de Imagens Georreferenciadas no Contexto do Mapeamento Terrestre Móvel. Presidente Prudente. Mestrado, dissertação. Curso de Pós Graduação em Ciências Cartográficas. Universidade Estadual Paulista. 2001. 74p.

OLIVEIRA, E. B. Implementação do módulo de interseção fotogramétrica em um banco de imagens georreferenciadas. Dissertação (Mestrado) Mestrado em Ciências Cartográficas, Unesp, Presidente Prudente, 2003. 112 p.

PENG, Z.-R.; TSOU, M.-H. Internet GIS: Distributed Geographic Information Services for the Internet and Wireless Networks. New Jersey: John Wiley \& Sons Ltd. 2003. 720 p.

SILVA, F.A; ARTERO, A.O; PAIVA, M.S.V; BARBOSA, R. L. ALPRS - A New Approach for License Plate Recognition Using the SIFT Algorithm. Signal \& Image Processing: An International Journal, v. 4, 2013. pp. 17-33

SILVA, J. F. C.; CAMARGO, P. O.; GALLIS, R. B.A. Development of a low-cost mobile mapping system: a south american experience. Photogrammetric Record, v. 18, n. 101, 2003. pp. 5-26,

SILVA, J. F. C.; CAMARGO, P. O.; OLIVEIRA, R. A.; REISS, M. L. L.; SILVA, R. A. C.; GUARDIA, M. C.; GALLIS, R. B. A. Mapeamento de Ruas com um Sistema Móvel de Mapeamento Digital. Revista Brasileira de Cartografia, v.53, 2001. pp. $82-91$.

SILVA, J. F. C.; OLIVEIRA, R. A.; GALLIS, R. B. A. Georreferenced Road Image Database. In: International Symposium on Mobile Mapping Technology, 3, Cairo. International Archives, ISPRS, CD-ROM, 2001. 8p.

TAO, C. V. Mobile Mapping. Technology for Road Network Data Acquisition. Journal of Geospatial Engineering, 2 (2) 2000, pp. 1-13

TANG, W.; SELWOOD, J. Connecting Our World: GIS Web Services. Redlands, CA, USA: ESRI Press, 2003. 180 p.

TOSCHI, I.; RODRÍGUEZ-GONZÁLVEZ, P.; REMONDINO, F.; MINTO, S.; ORLANDINI, S.; FULLER, A. Accuracy evaluation of a mobile mapping system with advanced statistical methods. The International Archives of Photogrammetry, Remote Sensing and Spatial Information Sciences, 40(5), 2015, p.245. 
YANG, C.; WONG, D.; YANG, R.; KAFATOS, M.; LI, Q. (2004) - Performanceimproving techniques in web-based GIS. International Journal of Geographical Information Science, Vol: 19. pp. 319-342.

Rev. Bras. de Cartografia, vol. 70, Edição Especial "XXVII Congresso Brasileiro de Cartografia”, 2018. pp. $1644-1663$. 